\title{
Low IL-6, IL-10, and TNF- $\alpha$ and High IL-13 Cytokine Levels Are Associated with Severe Hepatic Fibrosis in Schistosoma mansoni Chronically Exposed Individuals
}

\author{
Mable M. Mutengo $\mathbb{D}^{1},{ }^{1,2}$ Takafira Mduluza, ${ }^{3}$ Paul Kelly, ${ }^{4}$ James C. L. Mwansa, ${ }^{1,2}$ \\ Geoffrey Kwenda, ${ }^{5}$ Patrick Musonda, ${ }^{6}$ and James Chipeta ${ }^{7}$ \\ ${ }^{1}$ Department of Pathology and Microbiology, University Teaching Hospital, Lusaka, Zambia \\ ${ }^{2}$ University of Zambia, Lusaka, Zambia \\ ${ }^{3}$ Department of Biochemistry, University of Zimbabwe, Mount Pleasant, Harare, Zimbabwe \\ ${ }^{4}$ Department of Internal Medicine, School of Medicine, University of Zambia, Lusaka, Zambia \\ ${ }^{5}$ Department of Biomedical Sciences, School of Health Sciences, University of Zambia, Lusaka, Zambia \\ ${ }^{6}$ Department of Epidemiology and Biostatistics, School of Public Health, University of Zambia, Lusaka, Zambia \\ ${ }^{7}$ Department of Pediatrics and Child Health, School of Medicine, University of Zambia, Lusaka, Zambia
}

Correspondence should be addressed to Mable M. Mutengo; mmutengo@yahoo.com

Received 29 August 2017; Accepted 21 December 2017; Published 23 January 2018

Academic Editor: José F. Silveira

Copyright (C) 2018 Mable M. Mutengo et al. This is an open access article distributed under the Creative Commons Attribution License, which permits unrestricted use, distribution, and reproduction in any medium, provided the original work is properly cited.

\begin{abstract}
Several studies have attributed the etiopathogenesis of chronic Schistosoma mansoni related hepatic fibrosis to unregulated immune responses against trapped parasite ova in the host. However, there is limited data on immune profiles associated with varying degrees of the disease in a population under chronic exposure to the parasite. We therefore investigated the role of selected T-helper (Th)1, Th2, and Th17 cytokines in relation to hepatic fibrosis severity among individuals resident in a hyper-Schistosoma mansoni endemic region of Western Zambia. Two hundred and forty-four S. mansoni infected individuals with and without fibrosis were analysed for cytokine profiles. Based on hepatic fibrosis stage as determined by ultrasound, participants were categorized into Group 0, Group I, Group II, and Group III. Cytokines were measured in S. mansoni egg stimulated whole blood culture supernatants using the BD Cytometric Bead Array kits. Compared to the nonfibrotic group, participants in the severe hepatic fibrotic group produced less interleukin- (IL-) 6, IL-10, and tumour necrosis factor-alpha (TNF- $\alpha$ ). On the other hand, IL-13 was significantly elevated in this group compared to the nonfibrotic group $(p<0.001$ ). Our results suggest that low IL-6, IL-10, and TNF- $\alpha$ and high IL-13 levels may influence $S$. mansoni disease progression.
\end{abstract}

\section{Introduction}

In endemic areas, Schistosoma mansoni disease presentations are often subtle and unrecognized. However, in a subset of infected individuals, severe hepatic fibrosis occurs due to excessive deposition of extracellular matrices in portal spaces in response to trapped Schistosoma egg leading to presinusoidal fibrosis and portal hypertension $[1,2]$. In such individuals, complications such as splenomegaly, ascites, and esophageal varices that lead to life threatening haemorrhage may develop [1].
The pathology observed in S. mansoni disease is attributed to unregulated $\mathrm{CD} 4^{+}-\mathrm{T}$ cell immune responses directed against trapped parasite ova in the hepatic sinusoids [3]. Studies have demonstrated that $\mathrm{CD}^{+} \mathrm{T}$ helper (Th)1 and Th2 immune responses are responsible for the immunopathology observed in acute and chronic schistosomiasis [3, 4], respectively. As egg deposition builds up, Th1 immune responses are dampened and a more pronounced Th2 response is observed [5]. This process is characterized by a granulomatous reaction with subsequent fibrosis [6], which is dependent mainly on Th2 mediated 
cytokines including interleukin- (IL-) 4, IL-5, and IL-13 [79]. Among the Th2 cytokines involved in the pathogenesis of $S$. mansoni, IL-13 has been identified as a key profibrotic cytokine in the development of Schistosoma induced hepatic fibrosis, the main cause of morbidity and mortality in chronic S. mansoni disease [8-10].

While it has been previously reported that Th1 immune responses are associated with acute infection [5], studies are now suggesting that Thl cytokines such as IFN- $\gamma$ and TNF- $\alpha$ could be involved in regulating hepatic fibrosis observed in chronic schistosomiasis disease. IFN- $\gamma$ is a known antifibrogenic cytokine [11] that has been shown to confer protection against fibrosis in $S$. mansoni infected individuals $[12,13]$. IFN- $\gamma$ inhibits the synthesis of extracellular molecules by the hepatic stellate cells $[11,14]$ and enhances matrix metalloprotease (MMP) gene expression [15]. Low levels of this cytokine are reported to be associated with severe periportal fibrosis in S. mansoni infected people [2]. On the other hand the role of tumour necrosis factor-alpha (TNF- $\alpha$ ) in regulating schistosomiasis disease remains unclear. Whereas some studies have documented that TNF- $\alpha$ is protective against severe disease [16], high levels are shown to aggravate disease $[12,17,18]$. Recently, IL-17A, a Th17 cytokine, was shown to contribute to the immunopathogenesis of schistosomiasis in both human and experimental animal models [19, 20], perhaps by inducing the deposition of cellular and collagen substances in the liver [21]. However, data on whether IL$17 \mathrm{~A}$ is associated with severe $S$. mansoni hepatic disease in humans is limited. Furthermore, the role of Th1 and Th2 cytokines in different stages of $S$. mansoni disease in a population persistently exposed to Schistosome parasites remains understudied.

We have previously described hyperendemic foci of $S$. mansoni infection in Western Zambia [22]. In the current paper, we report findings on the role of selected Th1, Th2, Th17, and regulatory cytokines in individuals with varying stages of hepatic fibrosis in the same population.

\section{Materials and Methods}

2.1. Study Area and Population. The study population was randomly drawn from a $S$. mansoni hyperendemic region of Kaoma District in the Western province of Zambia as reported in detail elsewhere [23]. Participants positive for $S$. mansoni infection as determined by Kato-Katz or serology were recruited. Two hundred and fifty-five participants with varying stages of hepatic fibrosis were recruited into the study [23]. Eleven of the recruited participants were excluded from analysis as they did not meet the inclusion criteria. Due to limited community social infrastructure such as clean water supply and sanitation, residents are repeatedly exposed to $S$. mansoni parasites as they conduct their daily activities in the infested rivers. This study was approved by the University of Zambia Biomedical Research Ethics Committee (IRB00001131).

Based on ultrasound liver image pattern findings, $S$. mansoni infected participants were categorized into four groups: Group 0, infected with no fibrosis $(n=111)$; Group I, infected with mild fibrosis $(n=59)$; Group II, infected with moderate fibrosis $(n=43)$; and Group III, infected with severe fibrosis $(n=31)$. Prior to participation into the study, written informed consent and/or assent, where appropriate, was sought from potential participants. Participants with schistosomiasis were treated with $40 \mathrm{mg} / \mathrm{kg}$ body weight praziquantel while those with associated severe disease were referred to the nearest health facility for further management.

2.2. Parasitology and Serology for S. mansoni Infection. Stool samples from recruited participants were examined for the presence of $S$. mansoni infection by the Kato-Katz method as described previously [22]. Intensity of infection was classified based on the World Health Organization (WHO) criterion as light (1-99 epg), moderate (100-399 epg), and heavy ( $\geq 400$ epg) [24]. A serological Enzyme linked immunosorbent assay (ELISA) test for Schistosoma antibodies (Scimedx Corporation, Denville, NJ 07834, USA) was used to confirm exposure in participants with negative Kato-Katz readings. Absorbance of serum samples conjugated with Schistosoma antibodies was read on an EL-800 ELISA plate reader (BioTek Instruments Inc) and results were reported according to the kit manufacturer's instructions.

2.3. Ultrasound Evaluation for S. mansoni Related Morbidity. S. mansoni related hepatic morbidity was detected using a portable ultrasound machine (Mindray DP-1100 Plus electronic convex transducer: 35C50EB, 2.5/3.5/5.0 MHz, Shenzhen Mindray Bio-Medical Electronics Co., Ltd, Nanshan, Shenzhen 518057, China). Examinations were conducted by two ultrasound technicians, one of them having been trained in the detection of schistosomiasis-related morbidity under the Schistosomiasis Control Initiative Programme (SCI). The degree of hepatic fibrosis was defined based on the liver image patterns (IP) ranging from A to F [25]. Since these scores denote the degree of fibrosis, we therefore classified our participants into four groups as normal (A and B), mild fibrosis (C), moderate fibrosis (D), and severe fibrosis (E and F). Participants with IP unrelated to schistosomiasis graded $\mathrm{X}$ (fatty liver), $\mathrm{Y}$ (cirrhosis), and $\mathrm{Z}$ (undetermined) were excluded from analysis.

2.4. Stimulation of Whole Blood and Cytokine Analysis. Five milliliters of venous blood was collected from study participants into heparin vacutainers. The blood was diluted $1: 4$ with RPMI culture containing $2 \mathrm{~mm}$ L-glutamine, $100 \mathrm{u} / \mathrm{ml}$ penicillin, and $100 \mu \mathrm{g}$ streptomycin (Lonza Group, Ltd). The diluted blood was then transferred into 24 -well culture plates containing $10 \mu \mathrm{g} / \mathrm{ml}$ Schistosoma soluble egg antigen (SEA) (Theodor Bilharz Institute, Giza, Egypt) and was incubated at $37^{\circ} \mathrm{C}$ in the presence of $5 \%$ carbon dioxide for 24 hours. The culture supernatants were harvested into cryovials and stored initially at $-20^{\circ} \mathrm{C}$ and later transported on ice to the research laboratory in Lusaka, Zambia, where they were stored at $-80^{\circ} \mathrm{C}$ until required for analysis. To measure cytokine levels, the Human Th1/Th2/Th17 cytometric bead array (CBA) and the human IL-13 flex kits (BD Biosciences, San Jose, California) were used. The Th1/Th2/Th17 kit simultaneously detects IL-2, IL-4, IL-6, IL-10, TNF- $\alpha$, IFN- $\gamma$, and IL-17A cytokines in a single sample whereas the IL-13 flex kit detects IL-13. 
TABLE 1: Characteristics of study participants, by degree of hepatic fibrosis.

\begin{tabular}{|c|c|c|c|c|c|}
\hline Characteristics & $\begin{array}{l}\text { Group } 0 \\
n=111\end{array}$ & $\begin{array}{c}\text { Group I } \\
n=59\end{array}$ & $\begin{array}{c}\text { Group II } \\
n=43\end{array}$ & $\begin{array}{c}\text { Group III } \\
n=31\end{array}$ & $p$ value \\
\hline Median age, in yrs (IQR) & $28(16-42)$ & $29(17-40)$ & $38(34-46)$ & $39(28-46)$ & 0.00 \\
\hline \multicolumn{6}{|l|}{$\operatorname{Sex}, n(\%)$} \\
\hline Male & $44(50.6)$ & $21(24.1)$ & $15(17.2)$ & $7(8.1)$ & \\
\hline Female & $67(42.7)$ & $38(24.2)$ & $28(17.8)$ & $24(15.3)$ & 0.38 \\
\hline \multicolumn{6}{|l|}{${ }^{\mathrm{a}}$ Intensity of infection, $n(\%)$} \\
\hline Light & $29(46.0)$ & $17(27.0)$ & $10(15.8)$ & $7(11.1)$ & \\
\hline Moderate & $16(50.0)$ & $6(18.7)$ & $5(15.6)$ & $5(15.6)$ & \\
\hline Heavy & $14(73.7)$ & $3(15.8)$ & $2(10.5)$ & $0(0.0)$ & 0.43 \\
\hline $\mathrm{PVD}^{\mathrm{b}}$, mean \pm SEM & $10.16 \pm 0.23$ & $10.71 \pm 0.57$ & $14.32 \pm 0.57$ & $17.63 \pm 1.01$ & 0.00 \\
\hline
\end{tabular}

The groups were defined and categorized based on liver image pattern as stated in Materials and Methods. ${ }^{a}$ Intensity of infection is based on 114 S. mansoni ova positive participants and this was graded according to the WHO criteria [24]. ${ }^{\mathrm{b}} \mathrm{PVD}$ is the portal vein diameter.

TABLE 2: Median (interquartile range) cytokine levels $(\mathrm{pg} / \mathrm{ml})$ in the nonfibrotic and fibrotic groups.

\begin{tabular}{|c|c|c|c|}
\hline Cytokine & $\begin{array}{c}\text { No fibrosis } \\
n=111\end{array}$ & $\begin{array}{l}\text { Fibrosis } \\
n=133\end{array}$ & $p$ value \\
\hline IL-2 & $0.93(0,2.28)$ & $0.78(0,1.92)$ & 0.365 \\
\hline IL-4 & $0.20(0,1.11)$ & $0(0.0,0.88)$ & 0.264 \\
\hline IL-6 & $11208(7571,14714)$ & $8058(4146,11030)$ & $<0.001$ \\
\hline IL-10 & $139.6(76.80,239.20)$ & $77.7(32.0,128.70)$ & $<0.001$ \\
\hline IL-13 & $0.57(0.17,2.92)$ & $1.45(0.26,4.67)$ & $<0.001$ \\
\hline IL-17A & $4.59(1.7,10.0)$ & $6.64(3.18,12.63)$ & 0.05 \\
\hline IFN- $\gamma$ & $1.35(0.17,2.92)$ & $0.78(0.23,2.17)$ & 0.23 \\
\hline TNF- $\alpha$ & $722.2(404.8,1174.6)$ & $451.0(218.4,828.2)$ & $<0.001$ \\
\hline
\end{tabular}

The Mann-Whitney test was used to compare differences in median cytokine levels in individuals without fibrosis and those with fibrosis.

The assays were performed according to the manufacturer's instructions. Samples and standards were acquired on the BD FACsverse cytometer (BD Biosciences, San Jose, California) and the generated FSC files were analysed using FCAP Array version 3.0 software.

2.5. Statistical Analysis. The Shapiro-Wilkinson test was used to test for normality of the cytokine data. We used the Mann-Whitney test to determine differences in cytokine levels between the two groups and the Kruskal-Wallis test for differences among the four groups. Differences in cytokine measurements between the nonfibrotic group (Group 0 ) and each of the 3 groups (groups I, II, and III) were determined by Dunn's test for multiple comparisons. We used ANOVA to test for differences in continuous demographic variables while, for categorical variables, the chi-square test or the Fisher's exact test was used where appropriate. All statistical tests were two-sided and $p$ values $<0.05$ were considered significant. Statistical analysis was performed using Stata version 12 software (STATA Corporation; College Station, TX) and Graphpad prism 6 software (GraphPad Software, San Diego, USA).

\section{Results}

3.1. Characteristics of the Study Participants. The overall median age in this study population was 34 years (IQR = 19-42). As shown in Table 1, the median age difference across the four groups varied significantly $(p<0.001)$. Although there were more female than male participants, the difference between the groups was not significant $(p=0.38)$. In this study, no significant differences in intensity of infection were observed across the four groups $(p=0.43)$.

3.2. Cytokine Levels in the Different Stages of Portal Fibrosis. Cytokine levels in whole blood culture supernatants of 244 participants were determined in relation to fibrosis (Table 2). Median IL-6, IL-10, and TNF- $\alpha$ cytokine levels were significantly elevated in participants without fibrosis than those with fibrosis (Table 2).

On the other hand individuals with fibrosis produced more IL-13 than those without fibrosis. No statistical significance in IL-2, IL-4, IFN- $\gamma$, and IL-17A was observed between the two groups. A similar trend was observed when cytokines were analysed based on the degree of fibrosis (Figure 1). 


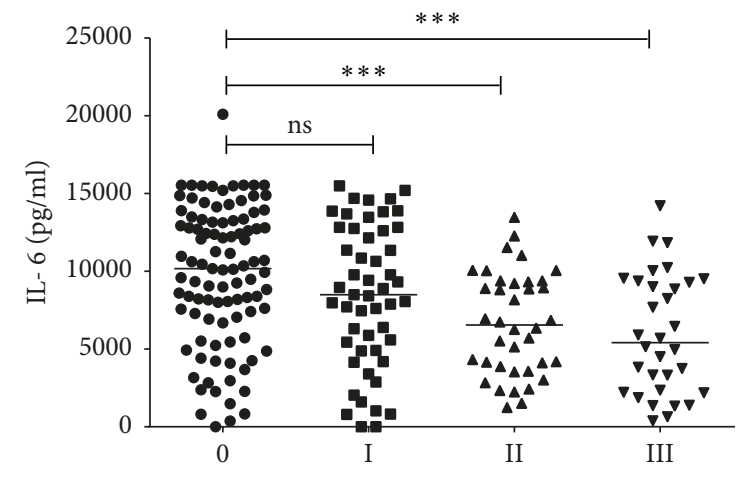

(a)

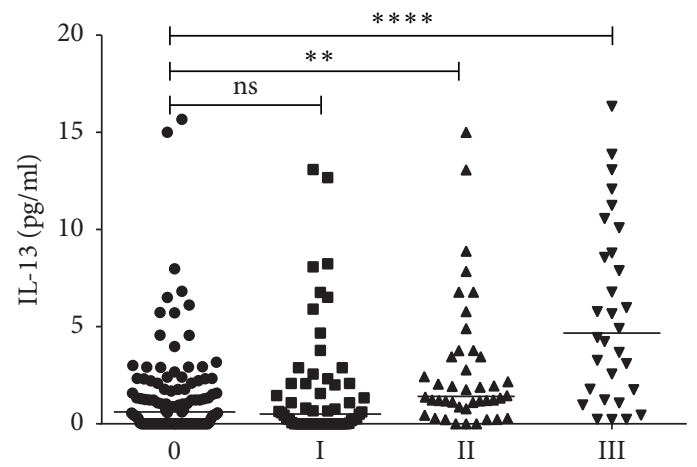

(c)

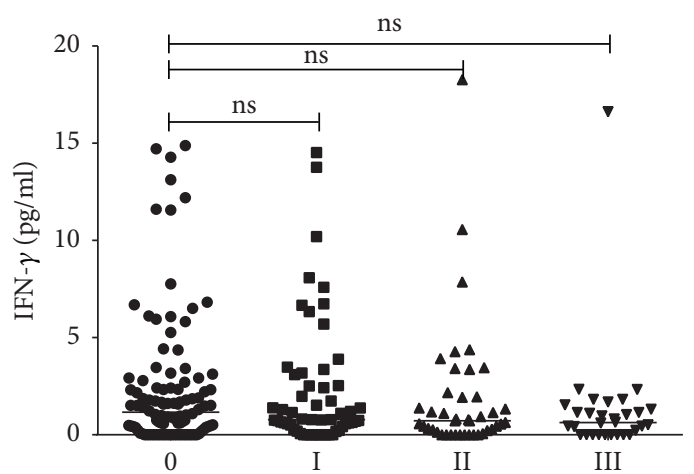

(e)

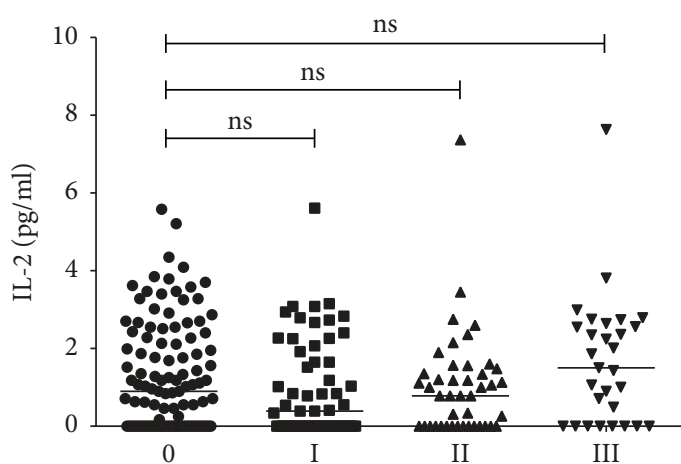

(g)

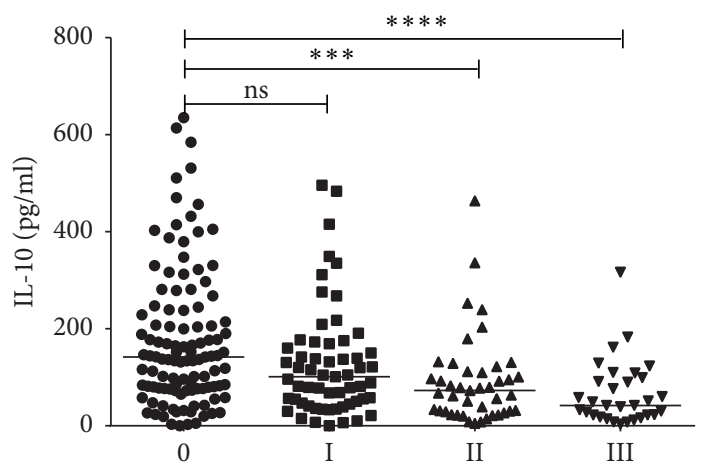

(b)

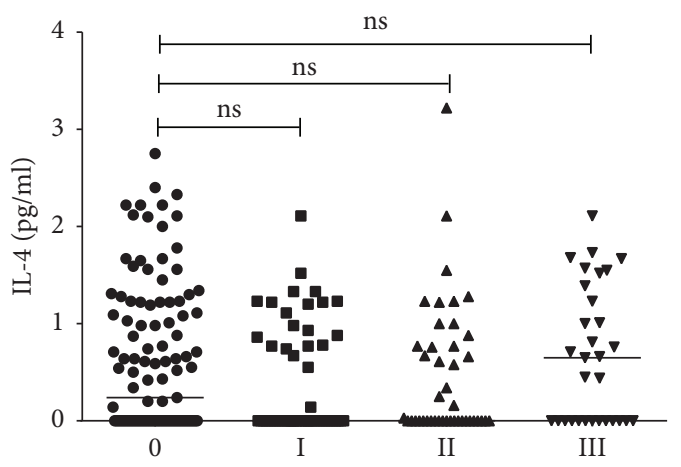

(d)

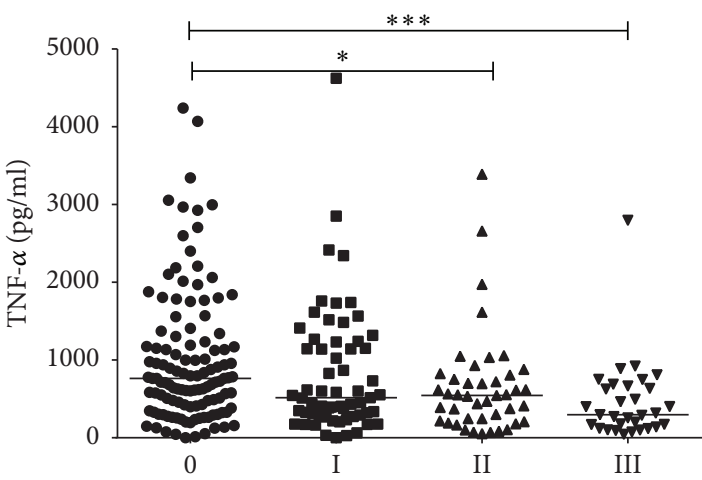

(f)

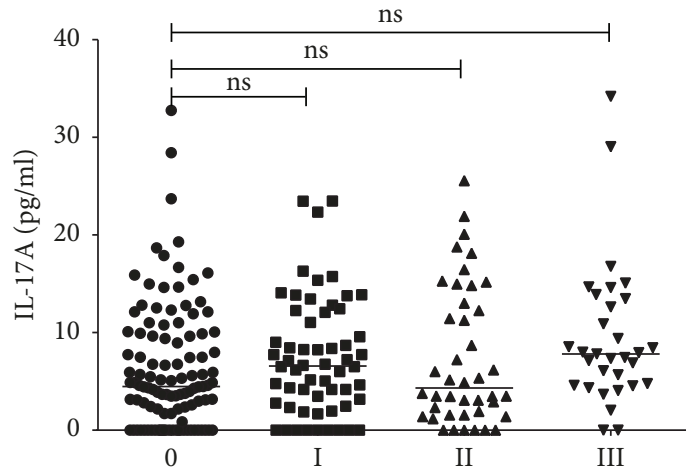

(h)

Figure 1: Cytokine levels in relation to degree of fibrosis. Median differences between Group 0 (control) and each of the three groups were determined using Dunn's test for multiple comparisons. Group 0 (infected with no fibrosis), Group I (infected with mild fibrosis), Group II (infected with moderate fibrosis), and Group III (infected with severe fibrosis). The significant level was set at $\leq 0.05$ for a two-sided test. ${ }^{*} p \leq 0.05,{ }^{* *} p \leq 0.01,{ }^{* * *} p \leq 0.001$, and ${ }^{* * * *} p \leq 0.0001$. 
Individuals in Group II and Group III produced less IL-6, IL-10, and TNF- $\alpha$ (Figures 1(a), 1(b), and 1(f)) than those in Group 0. Although not significant, participants in Group III had higher IL-17A levels than those in Group 0 (Figure 1(h)). In contrast, high IL-13 was strongly associated with severe fibrosis (Figure 1(c)).

\subsection{Cytokine Levels in the Nonfibrotic and Fibrotic Groups} According to Sex. To determine whether sex did influence cytokine expression, comparisons were made between female and male participants with and without fibrosis. In this analysis, IL-10 differed significantly between females and males in the nonfibrotic group (Figure 2(b)) whereas IL13 was high in males with fibrosis compared to females in the same group (Figure 2(c)). No statistically significant differences in the other measured cytokines were observed in the two groups.

\section{Discussion}

In the current study, we have observed that immune responses determined by the expression of Th1, Th2, Th17, and regulatory cytokines in response to Schistosoma egg antigen vary in each stage of $S$. mansoni disease. It appears that individuals expressing high levels of IL-6, IL-10, and TNF- $\alpha$ tend to be protected against severe hepatosplenic schistosomiasis. On the other hand, high levels of IL-13 correlated with disease severity. Furthermore, we found that the expression of most of these cytokines was not significantly influenced by sex. We further demonstrate for the first time the possible protective role of IL- 6 against severe hepatic fibrosis in a population chronically exposed to $S$. mansoni parasites.

Although hepatosplenic schistosomiasis is common in areas endemic for S. mansoni parasites, severe disease characterized by extended fibrosis leading to portal vein occlusion and subsequent hypertension, ascites, and formation of oesophageal varices occurs in a subset of individuals with the infection $[12,26]$. We have shown in our recent work that, among individuals with $S$. mansoni disease, less than $10 \%$ develop severe disease characterized with manifestations mentioned above [23]. The mechanisms surrounding the pathogenesis of S. mansoni hepatosplenic disease are centered on a well coordinated network of immune responses involving type 1 response in the early stages of the infection to a more intense type 2 response in the chronic egg deposition stage $[5,27]$.

Early studies had shown that Th2 associated cytokines (IL-4, IL-5, and IL-13) are the major drivers of pathology in S. mansoni disease $[4,5,28]$. In our study, IL-13 was significantly correlated with disease severity confirming earlier reports that this cytokine induces hepatic fibrosis $[29,30]$. The current findings are consistent with those from Brazil where a significant association between high IL-13 levels and severe hepatic fibrosis in S. mansoni infected individuals was documented [31]. On the contrary, no associations were found between this cytokine and the different stages of fibrosis in hepatosplenic patients with $S$. mansoni infection in another study [32]. Of interest in the present study was the possible protective role of IL- 6 against severe fibrosis. IL6 was highly expressed in individuals with no or mild fibrosis than in those with moderate/severe fibrosis in our study. The lack of IL-6 is associated with increased liver injury and enhanced activity of hepatic stellate cells resulting in fibrosis [33]. Recently, the therapeutic potential of IL-6 was shown by its ability to reverse liver fibrosis [34]. Increased levels in our study participants without fibrosis could suggest that IL-6 modulates $S$. mansoni related immune responses that could otherwise be harmful to the host. However, the actual mechanisms through which this cytokine could be involved in the immunopathogenesis of $S$. mansoni disease remain unclear. As IL-6 is highly pleiotypic, further studies may be needed to correlate it with respective gene activity in order to understand its protective mechanism.

Contrary to other studies that have documented an association between high levels of TNF- $\alpha$ with $S$. mansoni disease severity $[2,12,18]$, we observed elevated levels of this cytokine in participants with no or mild fibrosis. Our finding is in accordance with previous observations that have shown TNF- $\alpha$ to be protective against egg induced liver pathology $[16,35,36]$. In another study, TNF- $\alpha$ was only shown to be significantly associated with fibrosis when it was analysed together with IFN- $\gamma$ in a multivariate model [12]. This could mean that the pathogenic role of TNF- $\alpha$ is dependent on other cytokines such as IFN- $\gamma$. In a similar study, the effect of TNF- $\alpha$ on fibrosis was age and sex dependent. [2]. We, however, did not find any association between these two variables and TNF- $\alpha$ expression in relation to fibrosis.

Although we did not find statistically significant effect of IFN- $\gamma$ on hepatic fibrosis, individuals with severe fibrosis produced less of this cytokine than did those with no fibrosis. IFN- $\gamma$ inhibits the activity of hepatic stellate cells (HSC) which negatively affects the proliferation and synthesis of collagen and other extracellular matrices important for fibrosis $[11,14,37]$. Its lack of association with fibrosis in our study population could be attributed to the short incubation time of the SEA stimulated whole blood. Therefore, longer incubation times should be considered in future studies.

In the current study, low IL-10 levels were associated with moderate/severe hepatic fibrosis while IL-13 was raised in this group. This may indicate that, due to an ineffective regulatory immune response, there is excessive expression of IL-13 and subsequent uncontrolled deposition of extracellular matrix in the hepatic spaces resulting in fibrosis. The regulatory role of IL-10 has been well demonstrated. For instance, $S$. mansoni infected mice deficient in IL-10 show polarized immune responses which are detrimental to the survival of these animals $[38,39]$. Similarly, human participants with severe hepatic fibrosis tend to express less IL-10 compared to nonfibrotic individuals [2]. In our study, a linear relationship between IL-10 and the expression of IL- 6 and TNF- $\alpha$ in participants with mild or no fibrosis was observed. Consistent with the present findings, McGeachy and colleagues showed that IL-10 is upregulated in the presence of IL-6 [40]. It is plausible to suggest that these two cytokines act in synergy to modulate immune responses thus preventing the development of severe hepatic fibrosis. 


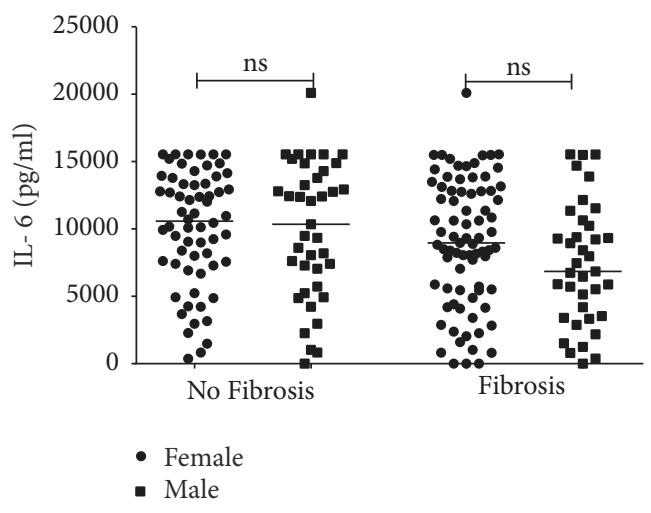

(a)

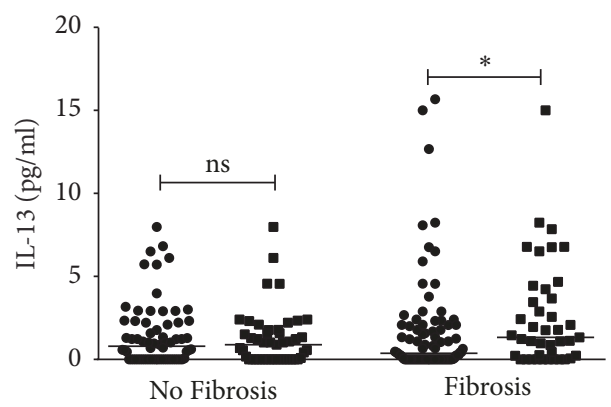

- Female

- Male

(c)

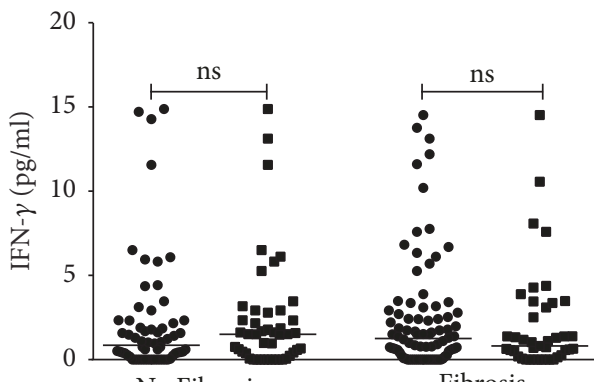

- Female

- Male

(e)

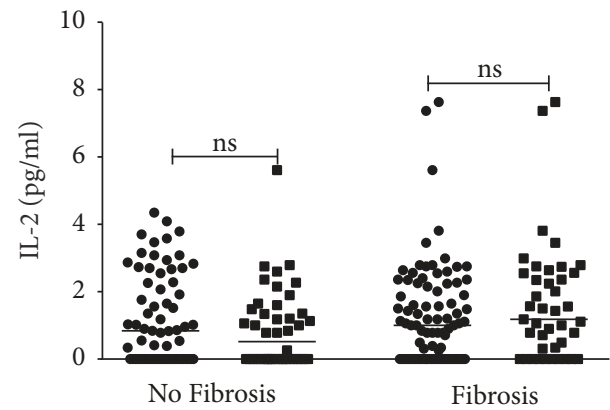

- Female

- Male

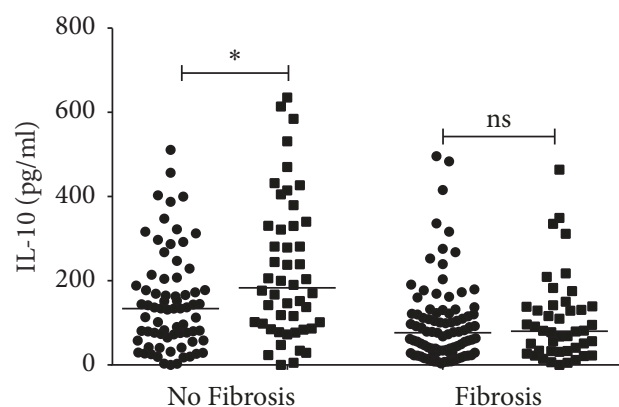

- Female

- Male

(b)

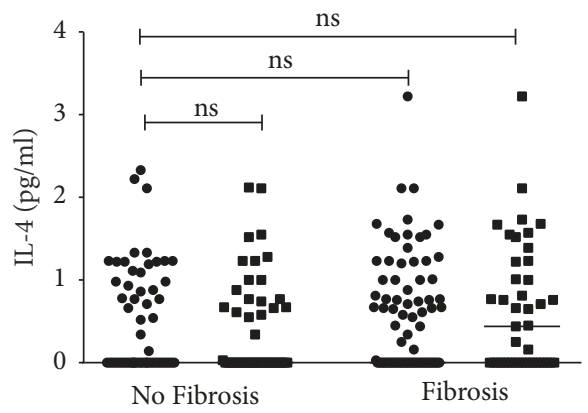

- Female

- Male

(d)

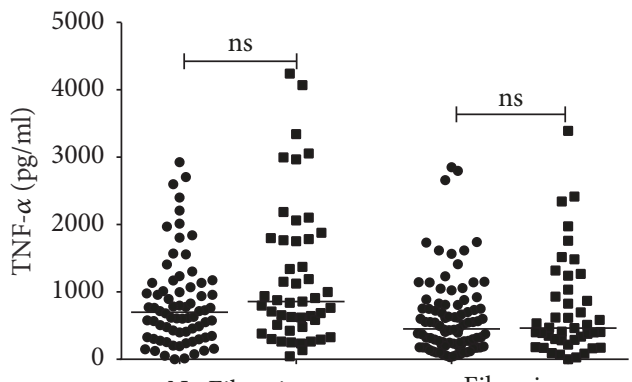

No Fibrosis

Fibrosis

- Female

- Male

(f)

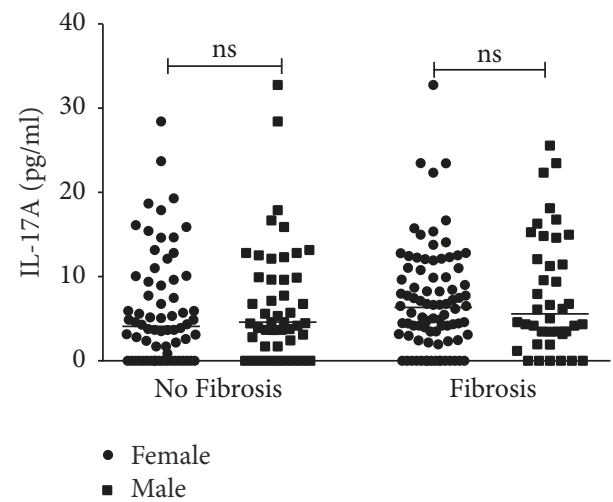

(h)

Figure 2: Cytokine Levels in female and male participants with or without fibrosis. Median values are shown by the horizontal line. The Mann-Whitney test was used to compare differences in median cytokine expression in individuals without fibrosis and those with fibrosis. ${ }^{*} p \leq 0.05$. 
To further understand whether Th17 $\mathrm{CD}^{+}-\mathrm{T}$ cells are involved in the pathogenesis of hepatic fibrosis, we investigated the role of IL-17A in relation to $S$. mansoni disease severity. Although a slight increase was observed in the severe hepatic fibrotic group compared to other groups, the difference was not statistically significant. IL-17A is one of the cytokines produced by the Th17 cells [41] and has been implicated in the pathogenesis of a number of disease conditions including the Schistosoma associated fibrosis [42].

Despite having well defined groups of hepatic fibrosis, the present study had no $S$. mansoni negative control group. A negative control group could have provided baseline data on the distribution of cytokines in uninfected individuals. In spite of this limitation, we were still able to draw conclusions based on the distinct cytokine profiles observed in the fibrotic groups in comparison to the nonfibrotic group.

\section{Conclusion}

In the present study, we have shown that high levels of IL6 , IL-10, and TNF- $\alpha$ could be protective against severe $S$. mansoni hepatic disease. On the other hand, our findings of high IL-13 in the severe fibrotic group are in agreement with previous studies suggesting that this cytokine is involved in fibrosis.

\section{Conflicts of Interest}

The authors declare no conflicts of interest.

\section{Authors' Contributions}

Mable M. Mutengo, Takafira Mduluza, Geoffrey Kwenda, and James Chipeta conceived and designed the experiments. Mable M. Mutengo performed the experiments. Mable M. Mutengo, Patrick Musonda, and Geoffrey Kwenda analysed the data. Paul Kelly contributed reagents/materials/analysis tools. Mable M. Mutengo, Takafira Mduluza, Paul Kelly, James C. L. Mwansa, Geoffrey Kwenda, Patrick Musonda, and James Chipeta wrote the paper. All authors read and approved the final manuscript.

\section{Acknowledgments}

The present work was funded by the Southern Africa Consortium for Research Excellence (SACORE) Wellcome Trust supported programme (Grant no. WT087537MA). The authors thank study participants from Kaoma district from whom the biological samples were obtained. They are also grateful to the Ministry of Health, Zambia (Kaoma district, Laboratory and Imaging Units) for giving them permission to conduct the study and also for the provision of transport and the ultrasound machine. They also acknowledge support received from the Department of Pathology and Microbiology (University Teaching Hospital) as well as the Tropical Gastroenterology (TROPGAN) lab. The invaluable support received from fellow SACORE and TROPGAN Ph.D. scholars within the University of Zambia-School of Medicine is acknowledged. All study support staff are acknowledged.

\section{References}

[1] J. Richter, J. G. Bode, D. Blondin et al., "Severe liver fibrosis caused by Schistosoma mansoni: Management and treatment with a transjugular intrahepatic portosystemic shunt," The Lancet Infectious Diseases, vol. 15, no. 6, pp. 731-737, 2015.

[2] M. Booth, J. K. Mwatha, S. Joseph et al., "Periportal fibrosis in human Schistosoma mansoni infection is associated with low IL-10, low IFN-gamma, high TNF-alpha, or low RANTES, depending on age and gender," The Journal of Immunology, vol. 172, no. 2, pp. 1295-1303, 2004.

[3] T. A. Wynn, R. W. Thompson, A. W. Cheever, and M. M. Mentink-Kane, "Immunopathogenesis of schistosomiasis," Immunological Reviews, vol. 201, pp. 156-167, 2004.

[4] M. H. Kaplan, J. R. Whitfield, D. L. Boros, and M. J. Grusby, “Th2 cells are required for the Schistosoma mansoni egg-induced granulomatous response," The Journal of Immunology, vol. 160, no. 4, pp. 1850-1856, 1998.

[5] E. J. Pearce, P. Caspar, J.-M. Grzych, F. A. Lewis, and A. Sher, "Downregulation of Thl cytokine production accompanies induction of Th2 responses by a parasitic helminth, Schistosoma mansoni," The Journal of Experimental Medicine, vol. 173, no. 1, pp. 159-166, 1991.

[6] E. J. Pearce and A. S. MacDonald, "The immunobiology of schistosomiasis," Nature Reviews Immunology, vol. 2, no. 7, pp. 499-511, 2002.

[7] C. Schwartz, K. Oeser, C. P. Da Costa, L. E. Layland, and D. Voehringer, "T cell-derived IL-4/IL-13 protects mice against fatal Schistosoma mansoni infection independently of basophils," The Journal of Immunology, vol. 193, no. 7, pp. 35903599, 2014.

[8] M. G. Chiaramonte, D. D. Donaldson, A. W. Cheever, and T. A. Wynn, "An IL-13 inhibitor blocks the development of hepatic fibrosis during a T-helper type 2-dominated inflammatory response," The Journal of Clinical Investigation, vol. 104, no. 6, pp. 777-785, 1999.

[9] A. R. De Jesus, A. Magalhães, D. Gonzalez Miranda et al., "Association of type 2 cytokines with hepatic fibrosis in human Schistosoma mansoni infection," Infection and Immunity, vol. 72, no. 6, pp. 3391-3397, 2004.

[10] P. G. Fallon, E. J. Richardson, G. J. McKenzie, and A. N. McKenzie, "Schistosome infection of transgenic mice defines distinct and contrasting pathogenic roles for IL- 4 and IL-13: IL13 is a profibrotic agent," The Journal of Immunology, vol. 164, no. 5, pp. 2585-2591, 2000.

[11] M. R. Duncan and B. Berman, " $\gamma$ interferon is the lymphokine and $\beta$ interferon the monokine responsible for inhibition of fibroblast collagen production and late but not early fibroblast proliferation," The Journal of Experimental Medicine, vol. 162, no. 2, pp. 516-527, 1985.

[12] S. Henri, C. Chevillard, A. Mergani et al., "Cytokine regulation of periportal fibrosis in humans infected with Schistosoma mansoni: IFN-gamma is associated with protection against fibrosis and TNF-alpha with aggravation of disease," The Journal of Immunology, vol. 169, no. 2, pp. 929-936, 2002.

[13] M. J. Czaja, F. R. Weiner, S. Takahashi et al., " $\gamma$-interferon treatment inhibits collagen deposition in murine schistosomiasis," Hepatology, vol. 10, no. 5, pp. 795-800, 1989.

[14] A. Mallat, A.-M. Preaux, S. Blazejewski, J. Rosenbaum, D. Dhumeaux, and P. Mavier, "Interferon alfa and gamma inhibit proliferation and collagen synthesis of human ito cells in culture," Hepatology, vol. 21, no. 4, pp. 1003-1010, 1995. 
[15] K. Tamai, H. Ishikawa, A. Mauviel, and J. Uitto, "Interferon- $\gamma$ coordinately upregulates matrix metalloprotease (MMP)- 1 and MMP-3, but not tissue inhibitor of metalloproteases (TIMP), expression in cultured keratinocytes," Journal of Investigative Dermatology, vol. 104, no. 3, pp. 384-390, 1995.

[16] K. F. Hoffmann, P. Caspar, A. W. Cheever, and T. A. Wynn, "IFN-gamma, IL-12, and TNF-alpha are required to maintain reduced liver pathology in mice vaccinated with Schistosoma mansoni eggs and IL-12," The Journal of Immunology, vol. 161, no. 8, pp. 4201-4210, 1998.

[17] A. N. Wamachi, J. S. Mayadev, P. L. Mungai et al., "Increased ratio of tumor necrosis factor- $\alpha$ to interleukin-10 production is associated with Schistosoma haematobium-induced urinarytract morbidity," The Journal of Infectious Diseases, vol. 190, no. 11, pp. 2020-2030, 2004.

[18] J. K. Mwatha, G. Kimani, T. Kamau et al., "High levels of TNF, soluble TNF receptors, soluble ICAM-1, and IFN-gamma, but low levels of IL-5, are associated with hepatosplenic disease in human schistosomiasis mansoni," The Journal of Immunology, vol. 160, no. 4, pp. 1992-1999, 1998.

[19] L. I. Rutitzky, J. R. Lopes da, Rosa., and M. J. Stadecker, "Severe CD4 T cell-mediated immunopathology in murine schistosomiasis is dependent on IL-12p40 and correlates with high levels of IL-17," The Journal of Immunology, vol. 175, no. 6, pp. 3920-3926, 2005.

[20] M. G. Shainheit, K. W. Lasocki, E. Finger et al., "The pathogenic Th17 cell response to major schistosome egg antigen is sequentially dependent on IL-23 and IL-1 $\beta$," The Journal of Immunology, vol. 187, no. 10, pp. 5328-5335, 2011.

[21] D. Chen, X. Luo, H. Xie, Z. Gao, H. Fang, and J. Huang, "Characteristics of IL-17 induction by Schistosoma japonicum infection in C57BL/6 mouse liver," The Journal of Immunology, vol. 139, no. 4, pp. 523-532, 2013.

[22] N. Katz, A. Chaves, and J. Pellegrino, "A simple device for quantitative stool thick smear technique in Schistosoma mansoni," Revista do Instituto de Medicina Tropical de São Paulo, vol. 14, pp. 397-400, 1972.

[23] M. M. Mutengo, J. C. L. Mwansa, T. Mduluza, S. Sianongo, and J. Chipeta, "High Schistosoma mansoni disease burden in a rural district of Western Zambia," The American Journal of Tropical Medicine and Hygiene, vol. 91, no. 5, pp. 965-972, 2014.

[24] WHO, Prevention and Control of Schistosomiasis and SoilTransmitted Helminthiasis, vol. 912 of World Health Organization Technical Report Series, 2002.

[25] J. Richter, C. Hatz, G. Campagne, N. R. Bergquist, and J. M. Jenkins, Ultrasound in Schistosomiasis. A practical Guide to the Standardized Use of Ultrasonogrpahy for the Assessment of Schistosomiasis Related Morbidity, World Health Organization, Geneva, Switzerland, 2000.

[26] M. Lubeya, C. Muloshi, K. S. Baboo, S. Sianongo, and P. Kelly, "Hepatosplenic schistosomiasis," The Lancet, vol. 376, no. 9753, p. $1645,2010$.

[27] J. M. Grzych, E. Pearce, A. Cheever et al., "Egg deposition is the major stimulus for the production of Th2 cytokines in murine schistosomiasis mansoni," The Journal of Immunology, vol. 146, no. 4, pp. 1322-1327, 1991.

[28] E. J. Pearce, A. Cheever, S. Leonard et al., "Schistosoma mansoni in IL-4-deficient mice," International Immunology, vol. 8, no. 4, pp. 435-444, 1996.

[29] Y. Liu, S. Munker, R. Müllenbach, and H.-L. Weng, "IL-13 signaling in liver fibrogenesis," Frontiers in Immunology, vol. 3, no. MAY, Article ID Article 116, 2012.
[30] R. Sugimoto, M. Enjoji, M. Nakamuta et al., "Effect of IL-4 and IL-13 on collagen production in cultured LI90 human hepatic stellate cells," Liver International, vol. 25, no. 2, pp. 420-428, 2005.

[31] L. F. Alves Oliveira, E. C. Moreno, G. Gazzinelli et al., "Cytokine production associated with periportal fibrosis during chronic schistosomiasis mansoni in humans," Infection and Immunity, vol. 74, no. 2, pp. 1215-1221, 2006.

[32] C. T. Brandt, M. Rino, M. G. Pitta, J. S. Muniz, O. Silveira Dde, and C. M. Castro, "Evaluation of the cytokines IL-10 and IL-13 as mediators in the progression of Symmers fibrosis in patients with hepatosplenic schistosomiasis mansoni," Revista do Colégio Brasileiro de Cirurgiões, vol. 37, no. 5, pp. 333-337, 2010.

[33] K. Kovalovich, R. A. Deangelis, W. Li, E. E. Furth, G. Ciliberto, and R. Taub, "Increased toxin-induced liver injury and fibrosis in interleukin-6- deficient mice," Hepatology, vol. 31, no. 1, pp. 149-159, 2000.

[34] G. A. Nasir, S. Mohsin, M. Khan et al., "Mesenchymal stem cells and Interleukin-6 attenuate liver fibrosis in mice," Journal of Translational Medicine, vol. 11, no. 1, article 78, 2013.

[35] S. J. Davies, K. C. Lim, R. B. Blank et al., "Involvement of TNF in limiting liver pathology and promoting parasite survival during schistosome infection," International Journal for Parasitology, vol. 34, no. 1, pp. 27-36, 2004.

[36] A. L. Joseph and D. L. Boros, "Tumor necrosis factor plays a role in Schistosoma mansoni egg-induced granulomatous inflammation," The Journal of Immunology, vol. 151, no. 10, pp. 5461-5471, 1993.

[37] D. C. Rockey and J. J. Chung, "Interferon gamma inhibits lipocyte activation and extracellular matrix mRNA expression during experimental liver injury: implications for treatment of hepatic fibrosis," Journal of Investigative Medicine, vol. 42, no. 4, pp. 660-670, 1994.

[38] K. F. Hoffmann, A. W. Cheever, and T. A. Wynn, "IL-10 and the dangers of immune polarization: excessive type 1 and type 2 cytokine responses induce distinct forms of lethal immunopathology in murine schistosomiasis," The Journal of Immunology, vol. 164, no. 12, pp. 6406-6416, 2000.

[39] M. Hesse, C. A. Piccirillo, Y. Belkaid et al., "The pathogenesis of schistosomiasis is controlled by cooperating IL-10-producing innate effector and regulatory T cells," The Journal of Immunology, vol. 172, no. 5, pp. 3157-3166, 2004.

[40] M. J. McGeachy, K. S. Bak-Jensen, Y. Chen et al., “TGF- $\beta$ and IL6 drive the production of IL-17 and IL-10 by T cells and restrain $\mathrm{T}_{H^{-}}-17$ cell-mediated pathology," Nature Immunology, vol. 8, no. 12, pp. 1390-1397, 2007.

[41] L. E. Harrington, R. D. Hatton, P. R. Mangan et al., "Interleukin 17-producing $\mathrm{CD}^{+}$effector $\mathrm{T}$ cells develop via a lineage distinct from the T helper type 1 and 2 lineages," Nature Immunology, vol. 6, no. 11, pp. 1123-1132, 2005.

[42] B. Wang, S. Liang, Y. Wang et al., "Th17 Down-regulation Is Involved in Reduced Progression of Schistosomiasis Fibrosis in ICOSL KO Mice," PLOS Neglected Tropical Diseases, vol. 9, no. $1,2015$. 


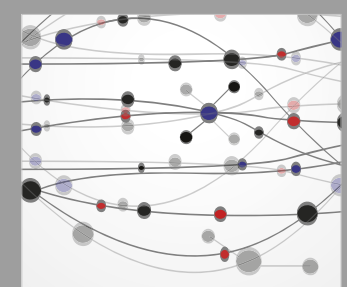

The Scientific World Journal
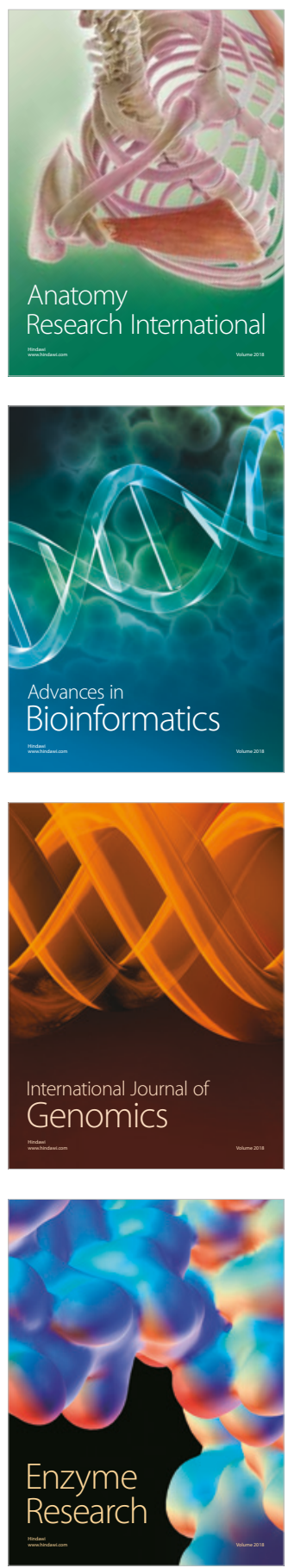
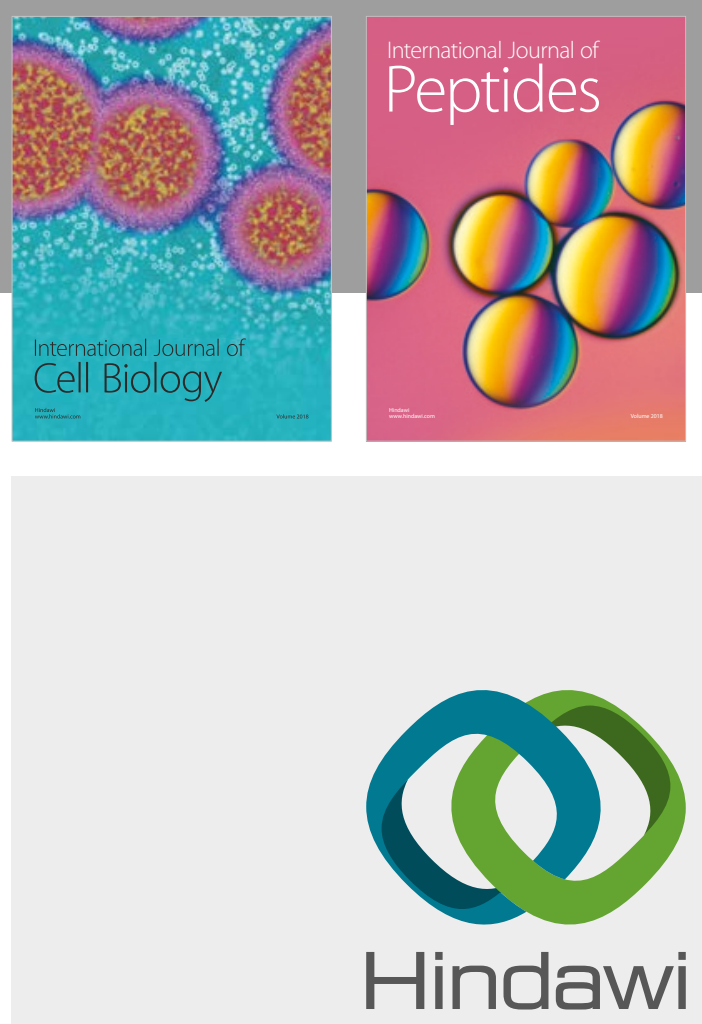

Submit your manuscripts at

www.hindawi.com
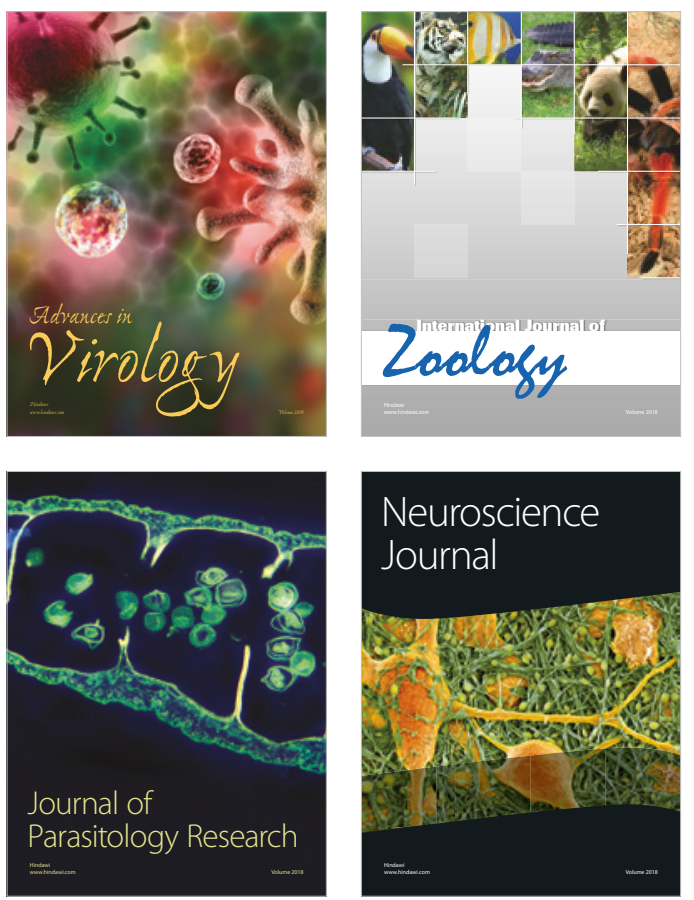
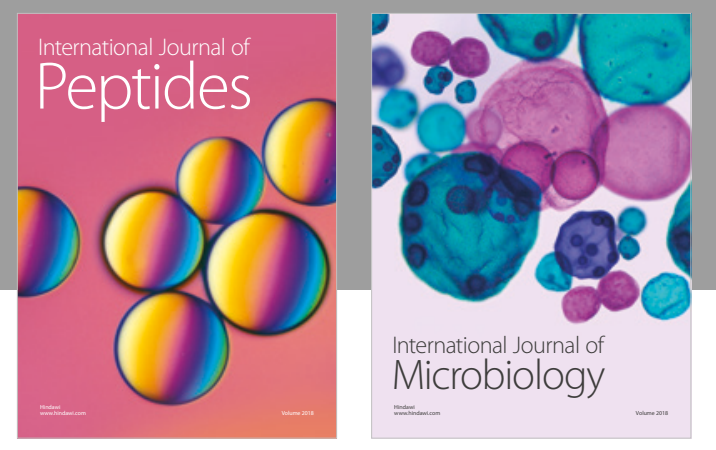

nternational Journal of Microbiology
Journal of
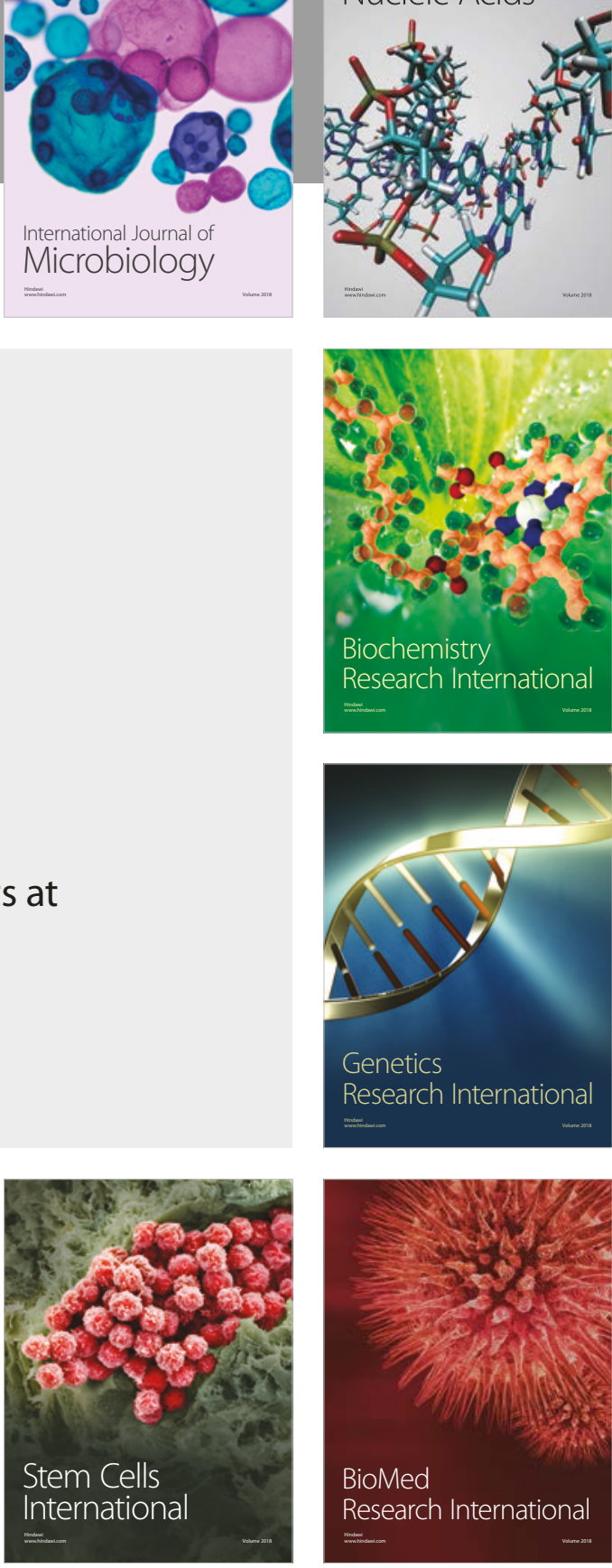
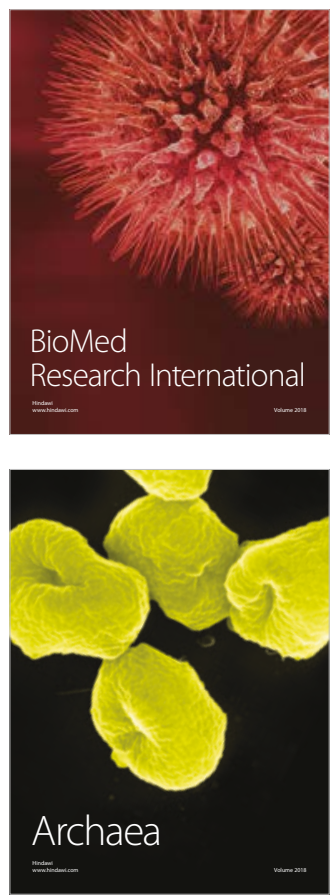